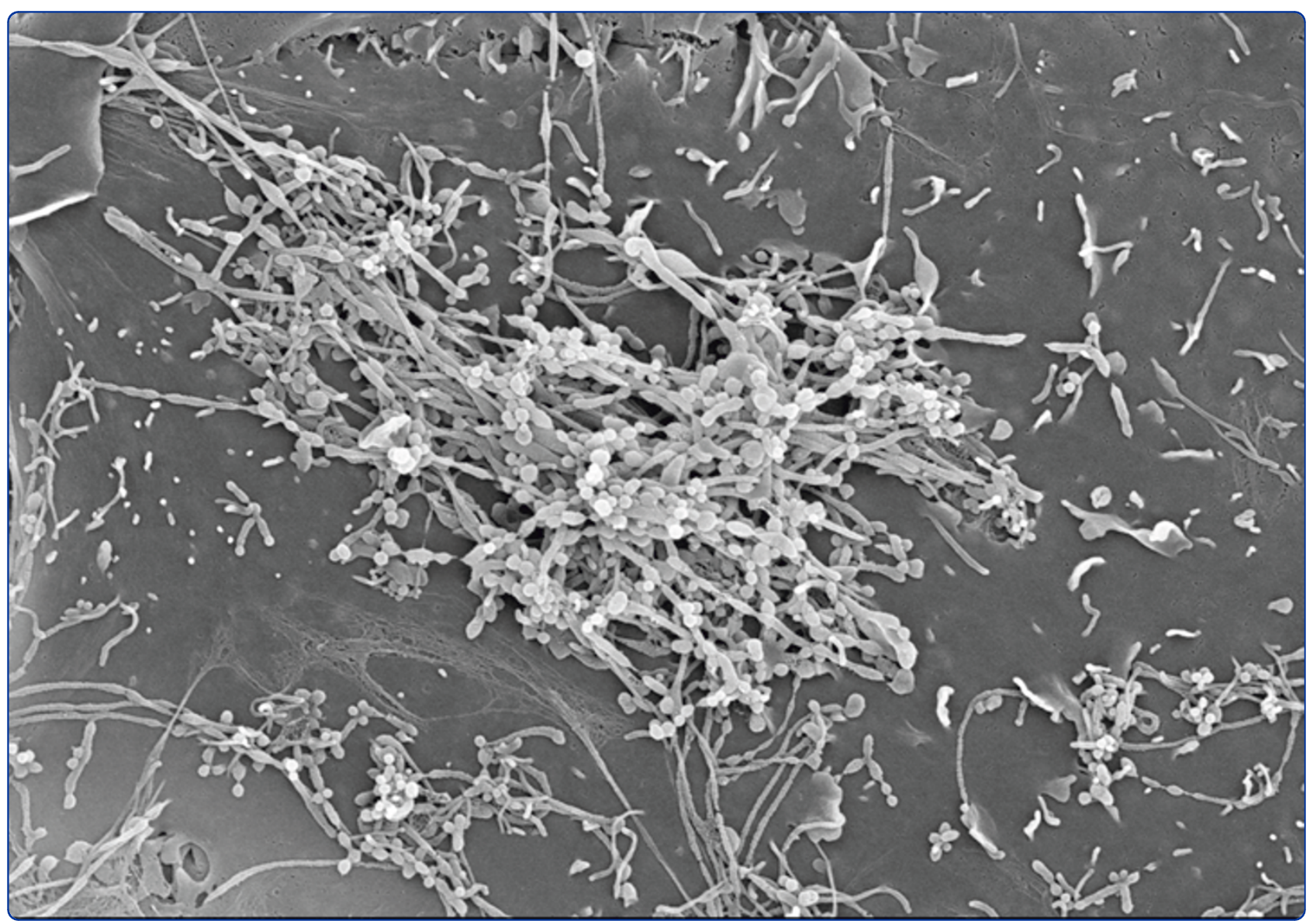

\title{
Bone marrow stromal cell antigen 2 (BST-2) restricts mouse mammary tumor virus (MMTV) replication in vivo
}

Jones et al. 


\title{
Bone marrow stromal cell antigen 2 (BST-2) restricts mouse mammary tumor virus (MMTV) replication in vivo
}

\author{
Philip H Jones, Harshini V Mehta, Martina Maric, Richard J Roller and Chioma M Okeoma*
}

\begin{abstract}
Background: Bone marrow stromal cell antigen 2 (BST-2) is a cellular factor that restricts the egress of viruses such as human immunodeficiency virus (HIV-1) from the surface of infected cells, preventing infection of new cells. BST2 is variably expressed in most cell types, and its expression is enhanced by cytokines such as type I interferon alpha (IFN- $\alpha$ ). In this present study, we used the beta-retrovirus, mouse mammary tumor virus (MMTV) as a model to examine the role of mouse BST-2 in host infection in vivo.
\end{abstract}

Results: By using RNA interference, we show that loss of BST-2 enhances MMTV replication in cultured mammary tumor cells and in vivo. In cultured cells, BST-2 inhibits virus accumulation in the culture medium, and co-localizes at the cell surface with virus structural proteins. Furthermore, both scanning electron micrograph (SEM) and transmission electron micrograph (TEM) show that MMTV accumulates on the surface of IFN $\alpha$-stimulated cells.

Conclusions: Our data provide evidence that BST-2 restricts MMTV release from naturally infected cells and that BST-2 is an antiviral factor in vivo.

Keywords: BST-2, Tetherin, Interferon alpha, MMTV, In vivo, SEM, TEM

\section{Background}

Bone marrow stromal cell antigen 2 (BST-2) protein also known as tetherin/CD317 is a potent restriction factor against a wide range of enveloped viruses such as HIV, FIV, KSHV, MMTV, SIV, Lassa, Marbug, Ebola, and MLV [1-5]. BST-2 achieves its anti-viral effect by connecting both viral and host cell membranes, thus preventing virus egress [6-9]. While BST-2 inhibits virus release, most viruses including HIV-1, HIV-2, and Ebola virus have developed strategies to antagonize BST-2 by degradation, down-regulation of expression, or reduction of its steady-state level $[1,2,7,10-14]$. In addition to inhibiting virus egress and virus replication in cell culture, there is evidence that, following interferon-induction, BST-2 is up-regulated and incorporated into budding virions $[2,6,8,9]$. While the antiviral activity of BST-2 has been demonstrated in tissue culture cells, there has been no evidence that BST-2 exerts antiviral activity in

\footnotetext{
*Correspondence: chioma-okeoma@uiowa.edu Department of Microbiology, University of lowa, Carver College of Medicine, lowa City, IA, USA
}

\section{Biomed Central}

(c) 2012 Jones et al; licensee BioMed Central Ltd. This is an Open Access article distributed under the terms of the Creative Commons Attribution License (http://creativecommons.org/licenses/by/2.0), which permits unrestricted use, distribution, and reproduction in any medium, provided the original work is properly cited. vivo. In this context, we evaluated the ability of mouse BST-2 to restrict the replication of the exogenous murine retrovirus mouse mammary tumor virus (MMTV) in cell culture and in mice.

In vivo, MMTV first infects antigen presenting cells (APCs) such as B cells and dendritic cells (DCs) at the site of infection [15-19]. MMTV infected APCs present virus-encoded superantigen ( $\mathrm{Sag}$ ) to $\mathrm{T}$ cells expressing Sag-specific T-cell receptor (TCR) V $\beta$ chains. This immunological synapse causes stimulation of Sag-reactive $\mathrm{T}$ cells and proliferation of lymphocytes thereby promoting virus replication. Both lymphoid and myeloid cells infected with MMTV are capable of producing infectious virus [19] and infected lymphoid cells are necessary for virus spread and mammary carcinogenesis $[20,21]$.

Although MMTV infects and causes mammary cancer in infected mice; nonetheless, infected cells do not produce high virus titer, and time to MMTV-induced cancer is rather long, suggesting that virus replication and spread may be restricted by host factors like BST-2. 
Indeed, we demonstrate that BST-2 co-localizes with MMTV Gag and Env, and inhibits MMTV particle release in tissue culture cells. Importantly, MMTV infection of mice was significantly inhibited by BST-2.

\section{Results \\ IFN $\alpha$ induces BST-2 expression and restricts MMTV release}

BST-2 expression results in retention of a wide range of virus-like particles (VLPs) assembled in tissue culture [1-4], and IFN $\alpha$ or IFN $\gamma$ treatment induces the transcription of the gene encoding BST-2 [1,22]. We hypothesized that IFNa induction of BST-2 in an MMTV producing cell line would enhance BST-2 expression and suppress MMTV release to the culture medium. We treated MMTV-producing GR cells with IFN $\alpha$ or vehicle and then measured BST-2 mRNA and surface protein levels, as well as extra- and intra- cellular viral particles. We observed a significant increase in BST-2 mRNA (Figure 1A) and protein (Figure 1B) levels upon IFN $\alpha$ treatment. This increase in BST-2 levels was accompanied by a reduction in the accumulation of MMTV particles in the culture supernatant, with no change in intracellular viral load as assessed by viral RNA (Figures 1C and 1D) and viral protein (Figure 1E). Additionally, the reduction in particle accumulation in culture supernatant results in lower viral load when such supernatants were used to infect MMTV susceptible cells (Figure 1F). This data demonstrate that IFN $\alpha$ induces endogenous BST-2 in MMTV infected cells and suggest that BST-2 may be the critical factor limiting the accumulation of MMTV particles in the culture supernatant.

Next, we examined whether BST-2 is responsible for IFN $\alpha$-dependent inhibition of MMTV release. GR cells stably silenced for BST-2 expression with a lentiviral construct carrying BST-2 specific shRNA or control shRNA were stimulated with IFN $\alpha$ or vehicle. Culture supernatants were collected 24 hours later and subjected to Western blot analysis for MMTV capsid protein $\mathrm{p} 27$. The corresponding cells were examined for BST-2 protein (Figure $1 G)$. Results show that while shRNA was able to silence BST-2 and enhance accumulation of viral particles in culture supernatant (Figure $1 \mathrm{H}$ ), IFN $\alpha$ stimulation of shRNA cells results in increased cell surface BST-2 protein expression (Figure 1G) and a reduction in the amount of viral particles accumulated in the culture supernatant (Figure $1 \mathrm{H}$ ). This result shows that the reduction in the amount of extracellular viral particles observed in IFN $\alpha$ treated cells is linked to BST-2 induction.

IFN $\alpha$ causes accumulation of MMTV structures at the cell surface

In IFN $\alpha$ treated cells, less MMTV particles were detected in the culture supernatant without apparent defect in accumulation of intracellular viral components (Figures 1E, 1H). As BST-2 was shown to block the release of various enveloped viruses/VLPs from the cells $[1,23]$, we hypothesize that exposure of MMTV-producing GR cells to IFN $\alpha$ will result in accumulation of MMTV virions on the cell surface. To test this, GR cells were stimulated with IFN $\alpha$ or vehicle and 24 hours later, cells were processed for scanning electron microscopy (SEM). In vehicle treated GR cells, $100 \mathrm{~nm}$ sized buds were readily observed (Figure $2 \mathrm{~A}$ ). The buds were commonly found on the tip of filamentous structures projecting from the cells surface. IFNo induction drastically increased the number of bud-containing filaments (Figure 2B). To confirm that the buds were indeed virions, thin sections of GR cells prepared under the same treatment conditions were analyzed with transmission electron microscope (TEM). Electron dense virion structures, about $100 \mathrm{~nm}$ in diameter (black arrowheads), were detected at the cells surface (Figure 2C and 2D). As expected, in IFN $\alpha$ treated cells, particles that are linked to each other were also observed (Figure 2D). The TEM thus validated that the filamentous structures observed with SEM contain virion(s). Together the electron microscopy data strongly suggest that exposure of MMTV infected cells to IFNa results in retention of MMTV virions on the cell surface.

\section{BST-2 co-localizes with MMTV structural proteins at the cell surface}

Consistent with its role in tethering virus particles to the surface of the infected cell, BST-2 has been shown to colocalize with structural proteins of other retroviruses along the plasma membrane of cells $[1,3]$. To examine whether BST-2 co-localizes with MMTV structural proteins, BST-2, rat glucocorticoid receptor (RSVGR), and MMTV proviral genome (HP) were co-transfected into $293 \mathrm{~T}$ cells. Two days later, cells were processed and immuno fluorescently stained to detect BST-2 and MMTV structural proteins. Results show partial co-localization of MMTV Env (Figure 3A) or Gag (Figure 3B) with BST-2 at the cell surface. Our data support previous demonstrations of co-localization of BST-2 with HIV-1 Env in infected cells [24] and co-localization of SIV Env and BST-2 in activated primary rhesus macaque lymphocytes infected with SIV [25]. In addition, our data are in line with previous report of BST-2 co-localization with the Gag proteins of other retro- and filo-viruses [3].

\section{Depletion of endogenous BST-2 enhances MMTV particle release and replication in cell culture}

To test the hypothesis that BST-2 is responsible for inhibition of MMTV release, we used an RNAi assay to determine whether silencing of endogenous BST-2 expression would lead to higher MMTV particle release and higher 

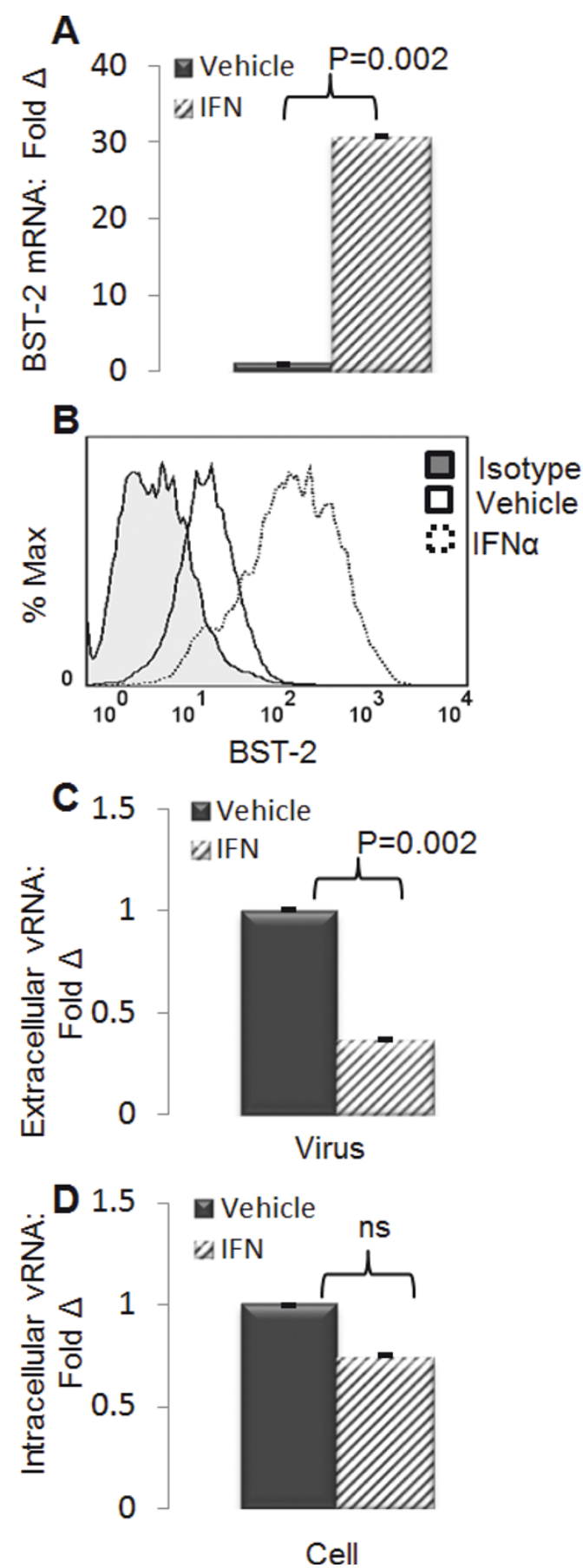

E
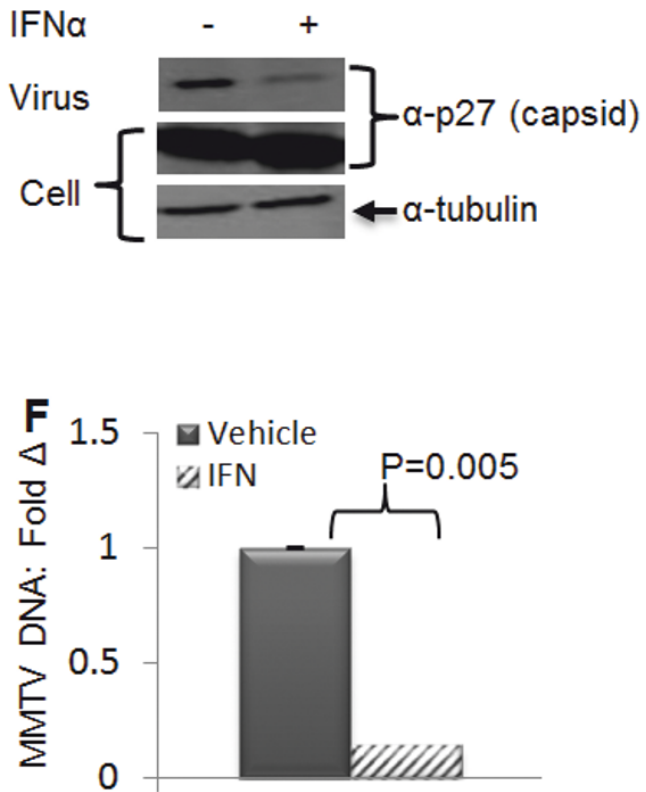

G

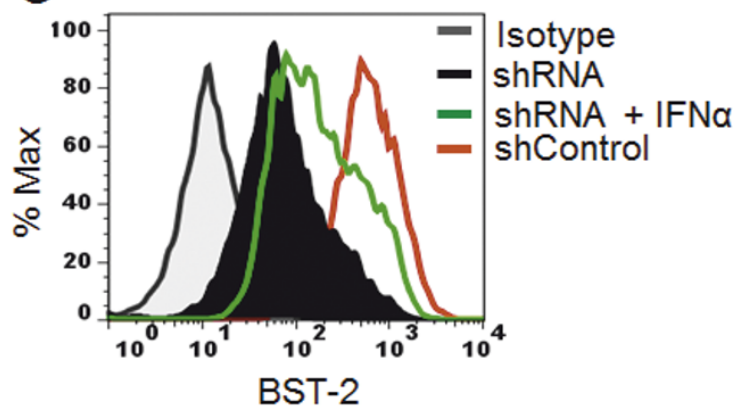

H

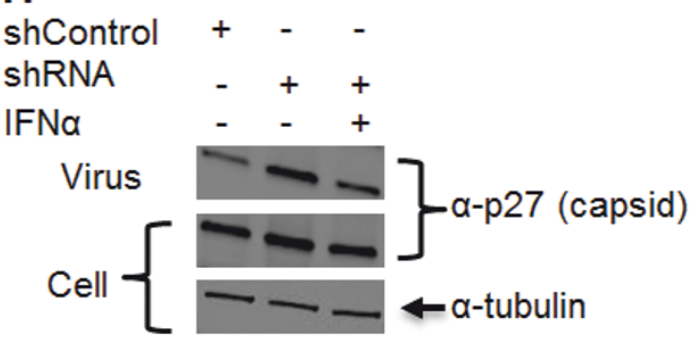

Figure 1 IFN $\alpha$-dependent induction of endogenous BST-2 restricts MMTV release. GR cells were treated with 1000 units of endotoxin free recombinant IFN $\alpha$ or PBS for 24 hours. Virus was purified from culture supernatants and the corresponding cell extracts were harvested. (A) Cell extracts were used to determine level of BST-2 mRNA by qPCR, presented as fold change in BST-2 mRNA relative to vehicle treated cells; (B) and surface BST-2 by flow cytometry with the peak on the left as the isotype antibody control. Culture supernatants and their corresponding cell extracts were used to evaluate level of viral RNA, (C) extracellular viral RNA, (D) intracellular viral RNA, (E) and viral protein detected by antibody to MMTV capsid protein p27. Tubulin is a loading control. Data are presented as fold change in vRNA relative to vehicle treated samples. (F) Culture supernatants from IFNa or vehicle treated GR cells were used to infect TRH3 cells. Twenty four hours after infection, cells were harvested and used for DNA extraction followed by qPCR analysis of viral DNA normalized to GAPDH. Data are presented as fold change in viral DNA of cells infected with supernatants from IFNa treated cells relative to viral DNA of cells infected with supernatants from vehicle treated cells. Error bars are standard deviation, and $\mathrm{p}$ is the significance level, ns is not significant. Experiments were performed at least three times with similar results. (G) GR cells were stably transduced with shRNA targeting mouse BST-2 gene (shRNA) or a non-targeting shRNA (shControl). Cells were treated with IFNa (1000 units/ml) or vehicle. Twenty four hours later a portion of cells were used for FACS analysis of surface BST-2 level. (H) Culture supernatant and the remaining cells were examined for level of extracellular and intracellular viral proteins by Western blot. 


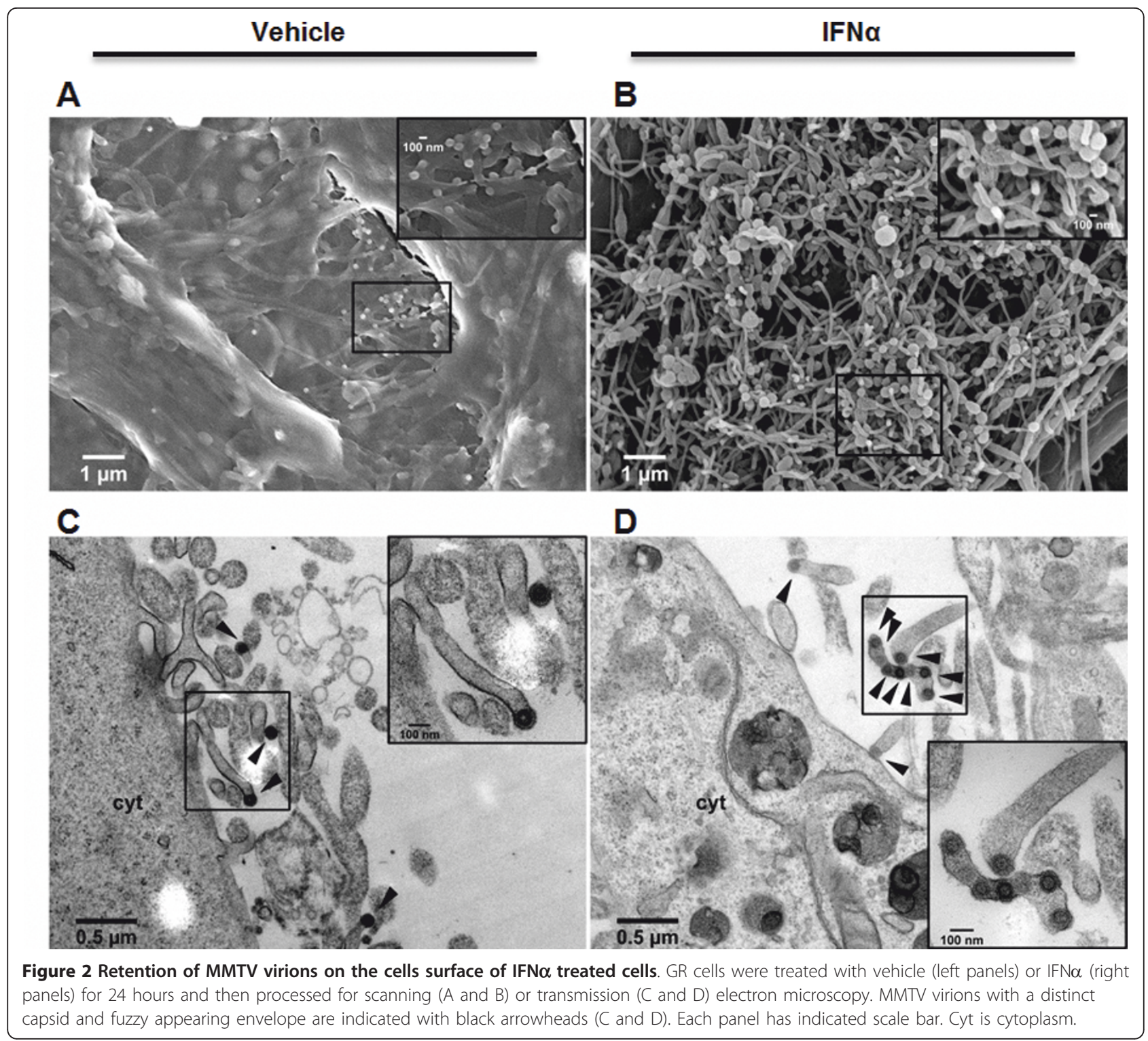

levels of infection. GR cells were transfected with either a control siRNA, or a siRNA that targets BST-2 mRNA (Figure 4A). Silencing endogenous BST-2 with siRNA (Figure 4B: upper panel - mRNA, lower panel - protein) enhanced extracellular viral RNA and protein yield compared to cells treated with control siRNA (Figure 4C and 4D respectively), suggesting that endogenous $\mathrm{BST}-2$ affects MMTV particle release. Similar results were obtained when endogenous BST-2 was silenced in GR cells with lentivirus-based shRNA targeting mouse BST-2 (Figures 4E,F,G). Given the ability of endogenous BST-2 to regulate MMTV release, we wondered if BST-2 expression could affect replication and spread of MMTV. Hence, we depleted BST-2 with siRNA in mammary epithelial NMuMG cells (Figure 4H: left panel -mRNA, right panel protein) and in 3T3 MEFs (Figure 4I: left panel - mRNA, right panel - protein). To evaluate the effect of reduced BST-2 levels on MMTV replication, we infected siRNA treated cells with MMTV, and observed significant differences in viral spread over a course of 96 hours in both NMuMG (Figure 4H: bottom panel) and 3T3 MEFs (Figure 4I: bottom panel). These data suggest that BST-2 impairs MMTV replication and spread in epithelial and fibroblast cells.

\section{Overexpression of BST-2 inhibits MMTV replication}

To confirm our finding that BST-2 restricts MMTV replication in cultured cells, we transiently expressed varying amounts of BST-2 or empty vector in TRH3 (293T cells that stably express MMTV entry receptor transferrin receptor 1). Twenty four hours later, a portion of cells were examined for BST-2 expression 


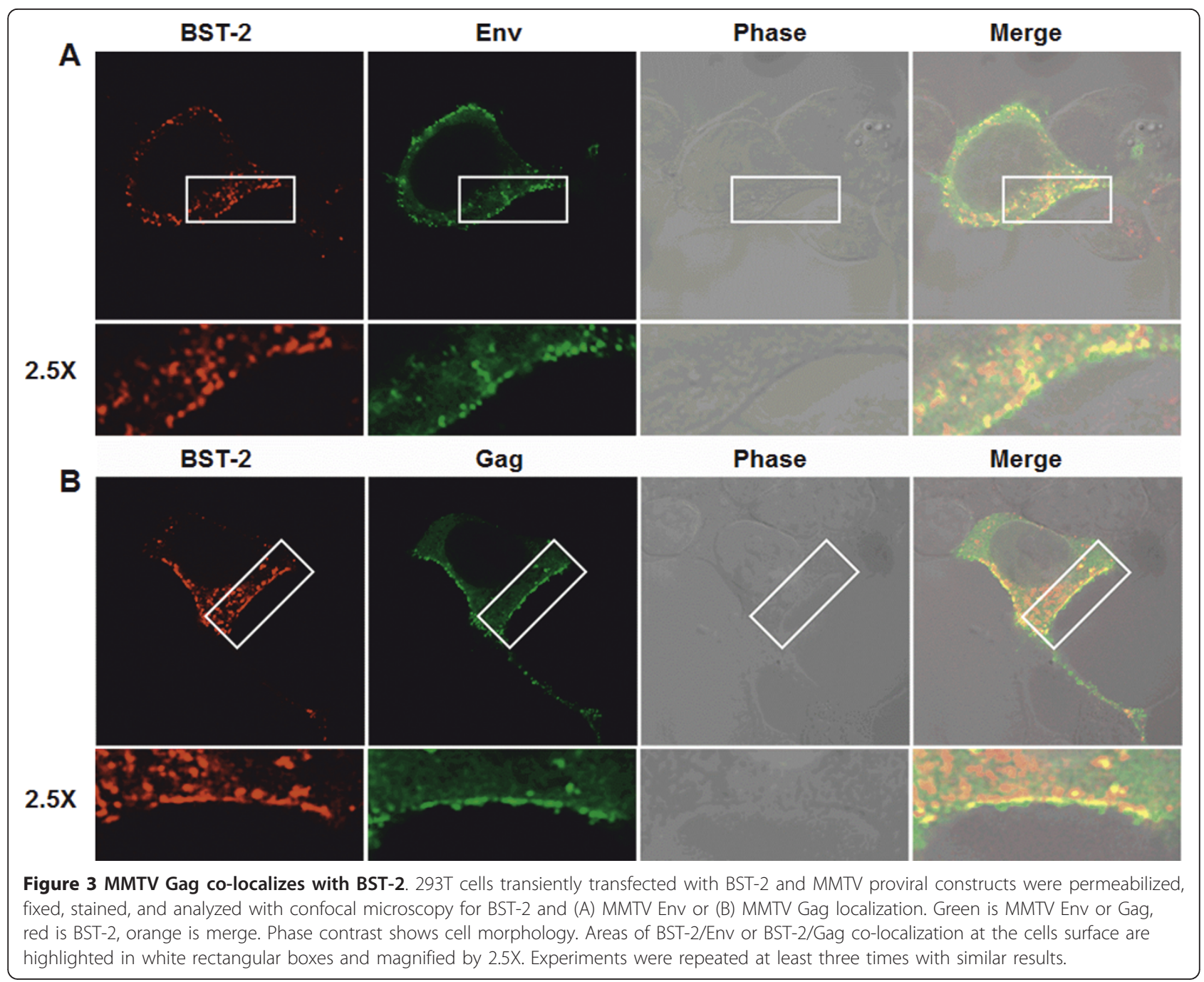

(Figure 5A), and the remaining cells were infected with MMTV. DNA was extracted from infected cells and used for qPCR examination of virus load 24 hours after infection. Results show that while cells transfected with BST-2 plasmid show lower levels of MMTV DNA across board, transfection of $100 \mathrm{ng}$ of BST-2 plasmid results in about $60 \%$ lower infection (Figure 5B), indicating that BST-2 restricts MMTV replication in a dose dependent manner. Whether lower infection observed in BST-2 transfected cells is a result of cells being infected at low multiplicity or lower number of cells being infected is unclear. To address this question, we first generated TRH3 cells stably expressing BST-2 or empty vector (Figure $5 \mathrm{C}$ ) and infected cells with MMTV.gfp purified from MMTV-producer cell line, CGRES6. These cells produce virions derived from a molecular clone of MMTV (pGR102ES) with a gfp gene inserted into its long terminal repeat (LTR) [26]. Infected cells were collected 24 and 96 hours later and examined for level and intensity of gfp expression. Results show that both at 24 and 96 hours after infection, level of gfp expression was significantly lower in BST-2 expressing cells compared to cells expressing empty vector control (Figure 5D). In addition, gfp mean fluorescence intensity (MFI) was also lower in cells expressing BST-2 at both time points (Figure 5E). These results suggest that BST2 potently restricts MMTV replication as evidenced by the larger proportion of cells infected in the absence of BST-2 (Figure 5D, solid blue line and solid red line) compared to lower number of cells infected in the presence of BST-2 (Figure 5D, broken blue line and broken red line).

\section{BST-2 specific siRNA depleted endogenous BST-2 in} murine lymph nodes

We have demonstrated that loss of BST-2 in tissue culture cells enhances MMTV replication and spread (Figures $4 \mathrm{H}$ and $4 \mathrm{I}$ lower panels). To examine the role of 


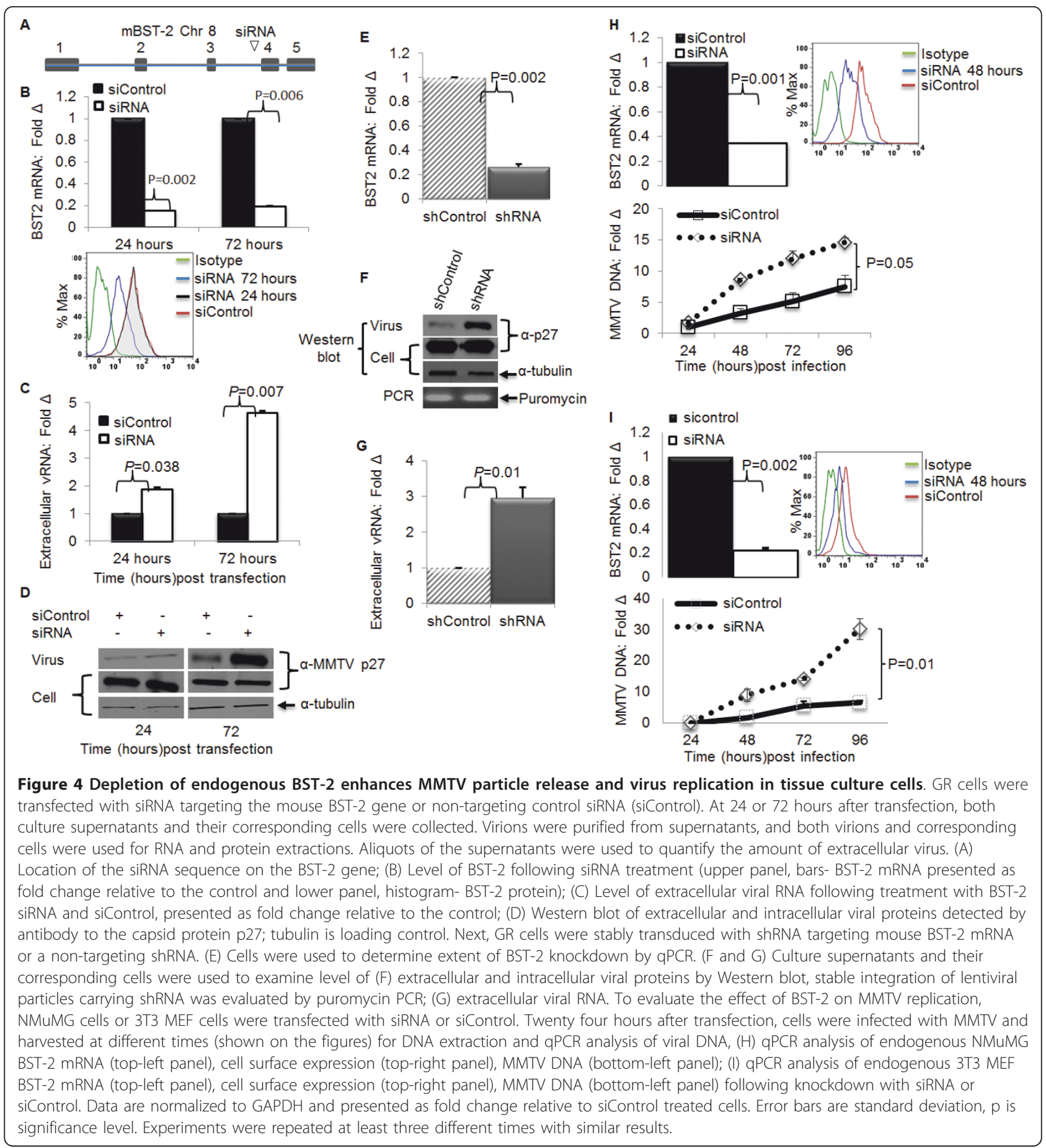

BST-2 in virus infection in vivo, we first determined whether MMTV target cells and tissues such as dendritic cells (DCs), macrophages (MФs), lymph nodes and spleens from different mouse strains $(\mathrm{C} 3 \mathrm{H} / \mathrm{HeN}$ and C57BL/6) that express BST-2 mRNA. We used the mammary epithelial cell line, NMuMG as a reference and show that BST-2 transcript is present in all MMTV targets (Figure 6A). BST-2 mRNA is higher in bone marrow derived DC (BMDC) compared to bone marrow derived macrophages (BMMФ), popliteal lymph nodes and the spleen, with a modest difference in BST-2 levels between $\mathrm{C} 3 \mathrm{H} / \mathrm{HeN}$ and $\mathrm{C} 57 \mathrm{BL} / 6$ mice. Since lymph nodes express BST-2, we examined whether BST-2 specific siRNA is capable of silencing BST-2 in vivo. Following inoculation of mice with siRNA or siControl subcutaneously in the hind footpad, we evaluated the 


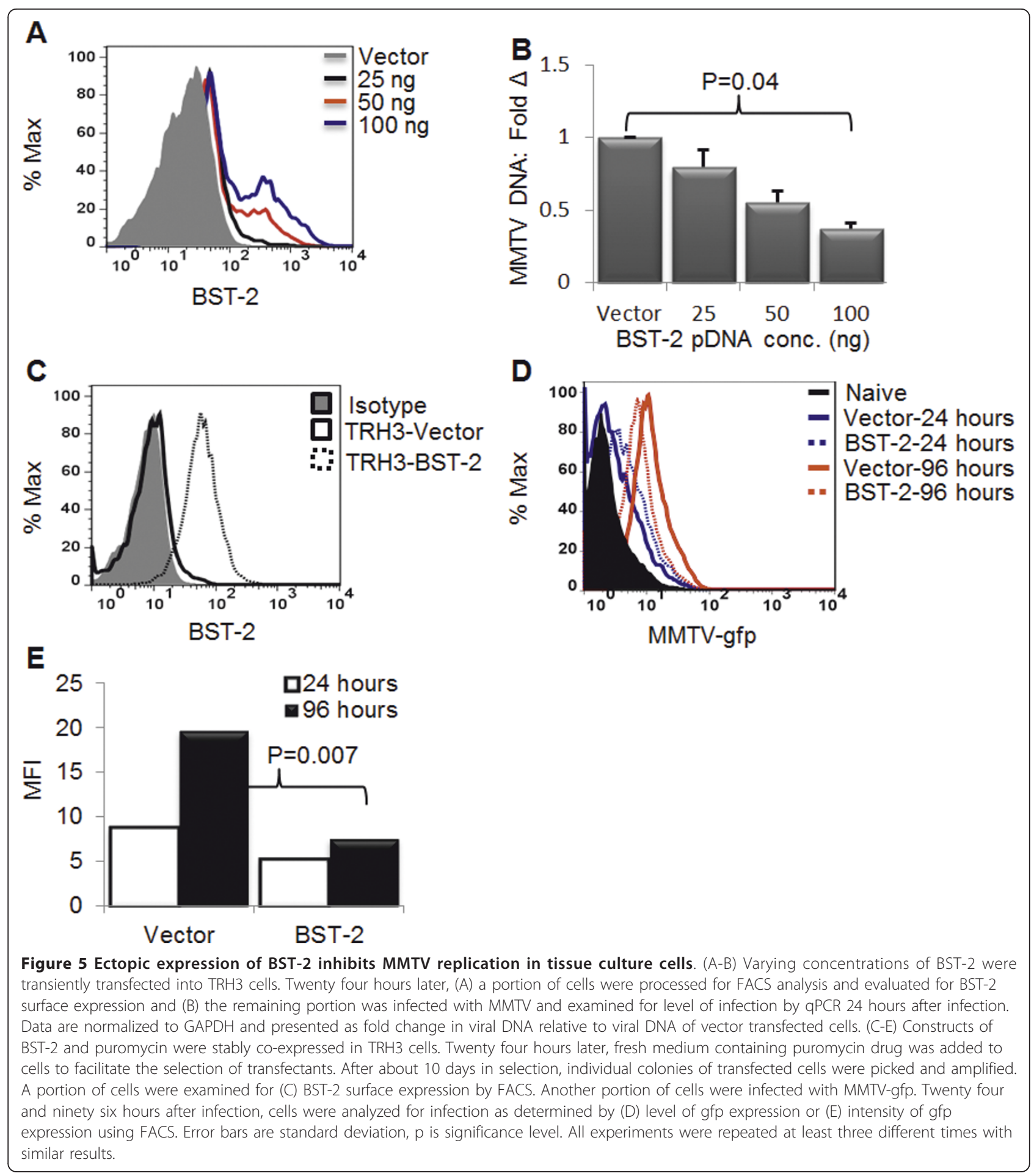

levels of BST-2 mRNA and protein and show that in 48 hours, siRNA significantly depleted lymph node BST-2 mRNA to about 7 fold (Figure 6B) with a modest reduction in protein level (Figure 6C: upper and lower), and no effect on BST-2 levels of mice inoculated with
siControl or no siRNA. We also examined the expression of IFN $\alpha$, IFN $\beta$, and 2'-5'-oligoadenylate synthetase (OAS) transcripts, and observed that mice treated with siRNA sequences have similar levels of IFN $\alpha$, IFN $\beta$, and OAS transcripts despite reduced BST-2 mRNA level 


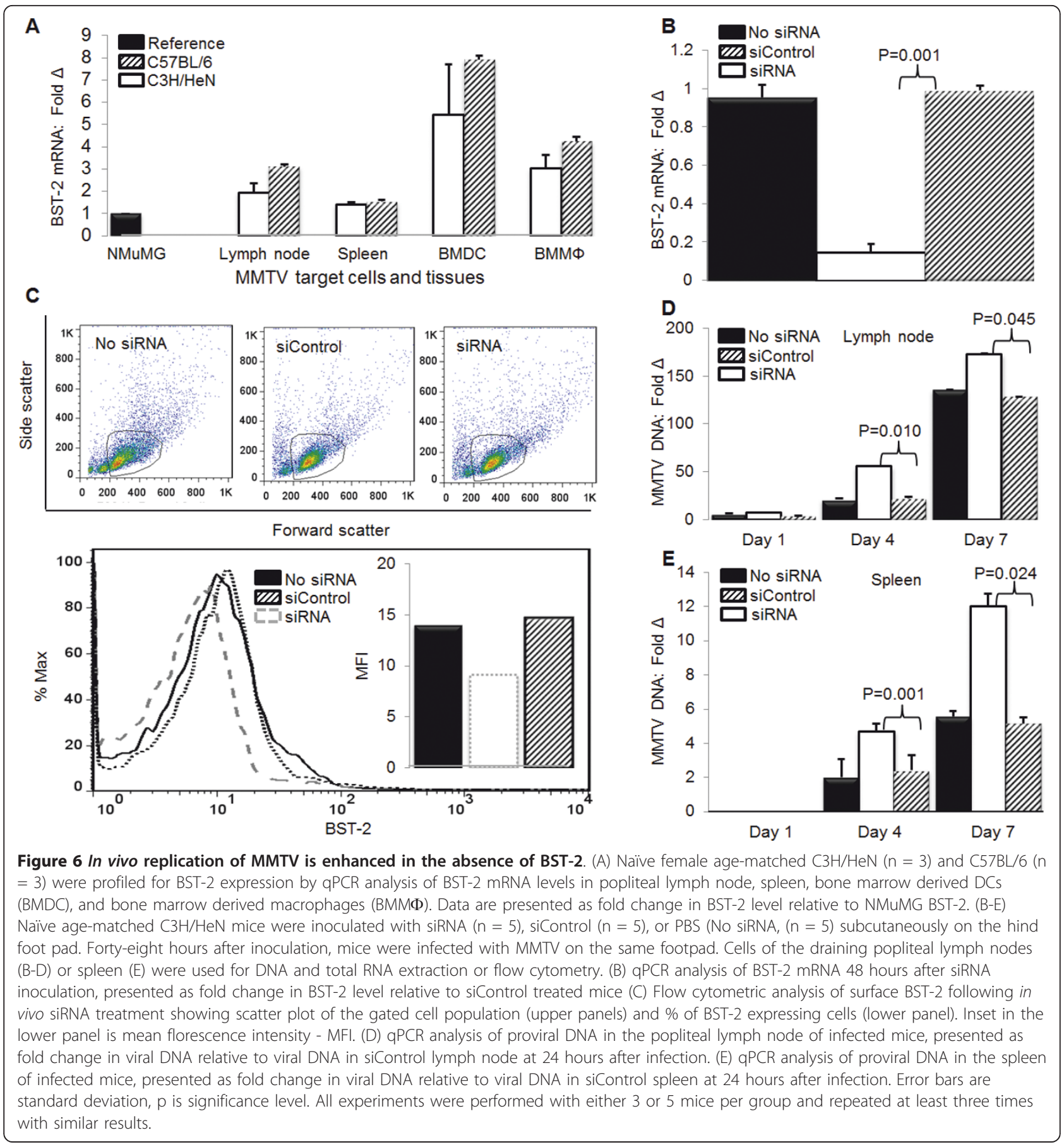

(Additional file 1). These data suggest that BST-2 specific siRNA reduced BST-2 expression in vivo without eliciting IFN response.

\section{siRNA mediated silencing of endogenous BST-2 enhances MMTV replication and spread in vivo}

Local administration of BST-2 siRNA mediates BST-2 depletion in vivo (Figures $6 \mathrm{~B}$ and $6 \mathrm{C}$ ). To determine the role of endogenous BST-2 in virus replication in vivo, we administered either a control siRNA, or siRNA that targets BST-2 mRNA to mice, followed by infection with MMTV. Virus replication was significantly higher in siRNA treated mice compared to their siControl or no siRNA counterparts (Figure 6D). In addition, we observed significantly higher virus load in the spleens of mice that received siRNA (Figure 6E). These data show for the first time that siRNA is capable of mediating BST-2 knock down in vivo (Figures $6 \mathrm{~B}$ and $6 \mathrm{C}$ ); and 
that loss of BST-2 in the draining lymph node for the site of initial infection results in higher rate of virus replication and dissemination in vivo (Figures 6D and $6 \mathrm{E})$.

\section{Discussion}

Here, we describe an important anti-viral function for BST-2 in the mouse. Many in vitro and ex vivo studies have shown that BST-2 inhibits the replication of a number of viruses [1-9]. By using MMTV, we directly demonstrate that BST-2 plays a role in virus replication in vivo. Administration of siRNA sequences into mouse footpad results in knock-down of endogenous lymph node BST-2. This assay allowed us to demonstrate that BST-2 functions as a virus restriction factor in a natural host organism. We chose to silence lymph node BST-2 because lymphocytes play a critical role in the in vivo infection with MMTV and other retroviruses such as HIV-1; and in the absence of a knockout model, siRNA provides the best alternative. The significantly higher virus load observed in BST-2 silenced lymph nodes implies that loss of BST-2 enhances virus replication.

BST-2 expression inhibits the egress of HIV-1, HIV-2, Ebola, Marburg and MLV; and we found that it also inhibits the accumulation of MMTV particles in the culture medium. The inhibitory effect of BST-2 on accumulation of extracellular MMTV particles in culture supernatant is consistent with the effect of BST-2 on virus release from the cell surface as has been observed for other viruses [1-5]. The observation that BST-2 colocalizes with MMTV Env and Gag further suggests that BST-2 prevents the accumulation of MMTV particles in culture supernatants. Our EM data support previously documented studies that MMTV buds off from the plasma membrane and microvilli [27]. The cell surface visualization by SEM provides an opportunity to demonstrate that induction of BST-2 with IFNa facilitates accumulation of MMTV on the cell surface. This finding was backed by our protein analyses showing that stimulation of MMTV producer cells with IFN $\alpha$ results in less accumulation of MMTV particles in the culture supernatant.

\section{Conclusion}

We have directly demonstrated in this study that loss of BST-2 enhances replication of MMTV in vivo. However, it remains to be determined why mice become infected with MMTV in the presence of the endogenous BST-2. It is worth noting that MMTV has co-existed with mice for over 20 million years [28] and perhaps must have evolved to avoid most hosts' anti-viral defense mechanisms. The findings of this study are important as it has shown that BST-2 function as anti-viral in the host. Based on this report, we are now focused on using
MMTV as a model to understand the role of BST-2 in viral pathogenesis.

\section{Materials and methods Ethics statement}

All experiments involving mice were performed in accordance to NIH guidelines, the Animal Welfare Act, and US federal law. The experiments approved by the University of Iowa Animal Care and Use Committee (IACUC). Mice were housed according to the policies of the Institutional Animal Care and Use Committee of the University of Iowa.

\section{Molecular constructs}

BST-2 plasmids used in the study were previously described [4] and kindly provided by Dr. Paul Bates, University of Pennsylvania, Philadelphia, PA. The MMTV env (Q61) construct [20], MMTV molecular clone HYB PRO (HP) [29], and the rat glucocorticoid receptor (RSVGR) [30] were kindly provided by Dr. Susan Ross, University of Pennsylvania, Philadelphia, PA.

\section{Animals}

$\mathrm{C} 57 \mathrm{BL} / 6$ and $\mathrm{C} 3 \mathrm{H} / \mathrm{HeN}$ mice were purchased from the Jackson Laboratory and National Cancer Institute (NCI) respectively.

\section{Cell lines}

NMuMG (derived from normal mammary tissue of a Namru mouse strain), GR (a mammary carcinoma cell line), Mm5MT (a mammary carcinoma cell line from $\mathrm{C} 3 \mathrm{H} / \mathrm{HeN}$ mouse strain), 3T3 MEF and 293T cell lines were purchased from American Type Culture Collection (ATCC). TRH3 cells are 293T-mTfR1 (293T cells that stably express MMTV entry receptor transferrin receptor 1 [mTfr1]) and have been previously described [31]. TRH3-BST-2 and TRH3-Vector cells (TRH3 cells stably expressing BST-2 or empty vector). CGRES6-GFP cells (feline kidney stably transfected with a GFP-tagged MMTV proviral construct [26] was obtained from Dr. Susan Ross of the university of Pennsylvania. All cells were maintained according to the suppliers' instructions. Both GR and Mm5MT cells are from MMTV-induced mammary tumor and both produce infectious MMTV particles.

\section{Generation of TRH3 cell-line stably expressing mBST-2}

To generate a TRH3-BST2 stable cell line, we co-transfected $6 \mu \mathrm{g}$ of BST-2 and $1 \mu \mathrm{g}$ of puromycin (selection marker) plasmids into TRH3 cells using polyethylenimine (PEI) transfection reagent. Following overnight incubation, transfection medium was changed, and replaced with medium containing puromycin dihydrochloride ( $3 \mu \mathrm{g} / \mathrm{ml}$, Santa Cruz Biotechnology, Inc.). 
The cells were left in culture with three changes of medium to remove dead cells. By day 10 after transfection, several individual clones of stably transfected cells emerged and clones were picked and expanded. Level of BST-2 expression was examined by FACs analysis. After examination, clones with the highest BST-2 expression were expanded and some cells frozen or used for experiments.

\section{Antibodies, siRNAs, shRNAs, and other reagents}

Antibodies used in this study includes goat anti-gp52 (Env) and anti-p27 (capsid) previously described [32,33]. Others are unconjugated rat anti-mouse BST-2 clone 129c, Alexa flour 647 conjugated anti-mouse BST-2 clone 129c [34], and FITC conjugated anti-mouse BST-2 clone 927 purchased from eBioscience. Rabbit anti-BST-2 and mouse anti-tubulin were from Abnova and Li-core respectively. The following isotype control antibodies were used: Rat IgG2a and Rat IgG2b, both from eBiosciences. The following secondary antibodies from Li-core were used: RDye 680 donkey anti-mouse, IRDye $680 \mathrm{LT}$ conjugated donkey anti-rabbit, IRDye $800 \mathrm{CW}$ donkey anti-goat, and IRDye $800 \mathrm{CW}$ conjugated goat anti-rabbit. Rabbit anti-rat Alexa Fluor 594 and chicken anti-goat Alexa Fluor 488 were from Invitrogen. The in vivo ready silencer Select target specific siRNA, control siRNA, and SiPort NeoFX transfection reagent were from Ambion while plasmids for target-specific Mission shRNA, control shRNA, and polybrene were obtained from Sigma. BST-2 shRNA and control shRNA lentiviral particles were produced from plasmids of target-specific Mission shRNA and control shRNA by the University of Iowa Vector Core Facility. Recombinant mouse interferon alpha (IFN- $\alpha$ ) was from Miltenyi Biotech. Vector-shield was from Vector Laboratories and Lipofectamine 2000 was from Invitrogen.

\section{Infection of mice}

In vivo infection of mice was performed with $50 \mu \mathrm{l}$ of MMTV.MM5MT inoculated subcutaneously on the hind footpad. At different times after infection, mice were sacrificed and the popliteal lymph node draining the site of infection from each mouse was harvested. Lymphocytes were obtained and used for fluorescenceactivated cell-sorting (FACS) analysis or used for DNA and RNA extraction.

\section{BST-2 knockdown by shRNA and siRNA in cell culture}

BST-2 shRNA lentiviral particles containing 3 targetspecific constructs and shRNA constructs encoding a scrambled sequence (Santa Cruz Biotechnology, Santa Cruz, CA) or target specific Mission shRNA and a corresponding control shRNA (Sigma) were used to knock down BST-2 gene expression. Briefly, mouse mammary epithelial tumor cell line GR were transduced with control or BST-2 shRNA lentiviral particles at a ratio of 5 infectious units of virus per cell in the presence of 8 $\mu \mathrm{g} / \mathrm{mL}$ polybrene. The next day, fresh media was added to cells and incubated for another 24 hours. In some experiments, puromycin at $2 \mu \mathrm{g} / \mathrm{ml}$ was added to the culture medium for the selection of cells that had stably incorporated shRNA. BST-2 gene silencing was confirmed at the mRNA and protein levels by qPCR and FACS. Knockdown of BST-2 by siRNA was achieved using Ambion's in vivo ready silencer Select target specific or siRNA in vivo ready scrambled siRNA. Briefly, 250 pico moles of siRNA sequences were mixed with Lipofectamine 2000 following manufacturer's recommendation and used to transfect NMuMG, GR or MEFs. The next day, fresh medium was added to cells and incubated until assayed for BST-2 and MMTV mRNA. NMuMG and MEFs were passaged and used for detection of BST-2 and virus replication experiments. Experiments were repeated at least 3 times with similar results.

\section{Western blots}

Western blots of virus preparations, cell lysates from MMTV infected cells, cells transiently transfected with env and capsid plasmids, or immuno-precipitates of protein complexes were probed with anti-total MMTV, anti-gp52, and anti-p27. The species-appropriate IRDye secondary antibodies were used, followed by detection with the Odyssey Infrared Imaging System (LI-COR Biosciences).

DNA, RNA isolation and real-time quantitative PCR (qPCR) DNA or total RNA was isolated from cells, tissues, and mice lymphocytes using the QIAGEN-QIAamp DNA Mini Kit or RNeasy Mini Kit (Qiagen, Inc.) respectively according to manufacturer's instructions. For cDNA synthesis, equivalent amounts of RNA treated with DNase I (Qiagen, Inc.) were reverse-transcribed with high capacity cDNA reverse transcription Kit (Applied Biosystems, $\mathrm{ABI}$ ), and the cDNA was amplified with primers specific to BST-2, MMTV.Mm5MT, IFN $\alpha$, IFN $\beta$, 2'-5'-OAS, and GAPDH. DNA was used to quantitatively detect integrated proviruses [32,33], using primers specific to the MMTV long terminal repeats and puromycin. Semi-quantitative PCR was performed using Veriti 96-Well Thermal Cycler, and real time qPCR was carried out using ABI 7500 FAST thermal cycler (ABI). All amplifications were normalized to GAPDH and data are presented as fold change relative WT or naïve sample depending on experiment. Sequence of primer pairs used is available (Table 1).

\section{Immuno-fluorescence and confocal microscopy}

293T cells were co-transfected with plasmids expressing BST-2, rat glucocorticoid receptor (RSVGR) and 
Table 1 Primer sequences

\begin{tabular}{|c|c|c|}
\hline Primer name & Forward sequence & Reverse sequence \\
\hline mBST-2 & TCAGGAGTCCCTGGAGAAGA & ATGGAGCTGCCAGAGTTCAC \\
\hline MMTV & CGTGAAAGACTCGCCAGAGCTA & TAATGTTCTATTAGTCCAGCCACTGT $[32,33]^{a}$ \\
\hline$\overline{\text { GAPDH }}$ & CCCCTTCATTGACCTCAACTACA & CGCTCCTGGAGGATGGTGAT $[32,33]^{a}$ \\
\hline Puromycin & CGTACGCACCCTCGCCGC & TCGTCCGCGACCCACACC \\
\hline IFNa & GCTAGGCTCTGTGCTTTCCTGATG & CTCAGGTACACAGTGATCCTGTGG \\
\hline IFN $\beta$ & AACAGGTGGATCCTCCACGCTGCG & GTGGAGAGCAGTTGAGGACATCTCC \\
\hline $2^{\prime}-5^{\prime}-\mathrm{OAS}$ & CTITGATGTCCTGGGTCATGT & CTCCGTGAAGCAGGTAGAG \\
\hline
\end{tabular}

Sequence of primer pairs used in this study. Citation means the primer has been previously reported.

MMTV proviral genome (HP) using lipofectamine 2000. The next day cells were treated with DEX and 24 hours later fixed in $4 \%$ paraformaldehyde in PBS for $10 \mathrm{~min}$ utes. Cells were then washed once with PBS and incubated for 3 hours in IF buffer $(0.5 \%$ Triton X-100, 0.5\%, sodium deoxycholate, $1 \%$ bovine serum albumin, $0.05 \%$ sodium azide in PSB). Primary antibodies were diluted in IF buffer as follows: rat anti-BST-2 (1:100), goat antip27 (1:100) or goat anti-gp52 (1:100) and detected with rabbit anti-rat Alexa Fluor 594 and chicken anti-goat Alexa Fluor 488. All secondary autobodies were dilated 1:1000 in IF buffer. All confocal microscopy images were taken with Zeiss 510 confocal microscope.

\section{Flow cytometry}

Approximately, $1 \times 10^{6}$ GR, MM5MT, NMuMG, 3T3 MEF, 293T, and lymphocytes were stained in PBS + $1 \%$ bovine serum albumin (Sigma-Aldrich) for $30 \mathrm{~min}$ on ice with Alexa flour 647 anti-mouse BST-2 (clone 129c) or FITC anti-mouse BST-2 (clone 927) and APC anti-mouse IgG2b. Cells were extensively washed in PBS, fixed with $2 \%$ paraformaldehyde and subjected to FACS. At least ten-thousand events were collected for each sample using FACS calibur flow cytometer (BD). Cellular frequency and mean fluorescence intensity (MFI) were determined by Flowjo analysis software (TreeStar).

\section{Virus release assay}

GR cells were induced for virus production with $1 \mu \mathrm{M}$ of dexamethasone (DEX) [35] and treated with IFN $\alpha$ or vehicle for 24 hours. Culture supernatants from cells were clarified by low speed centrifugation, passed through a $0.45 \mu \mathrm{m}$ filter, and virions were pelleted through a $20 \%$ sucrose layer at $32,000 \mathrm{~g}$ as previously described (30-31). Pellets of virus particles and corresponding cell extracts were denatured and analyzed by SDS-PAGE and Western blot assays. In some experiments, virus particles were used for $\mathrm{qPCR}$ analysis of viral RNA or to infect TRH3 cells.

\section{Statistical analysis}

Statistical analysis of significant differences between experimental groups was tested using paired two-tailed Student's t test, and a p value of 0.05 was considered significant. Error bars represent standard deviations.

\section{Additional material}

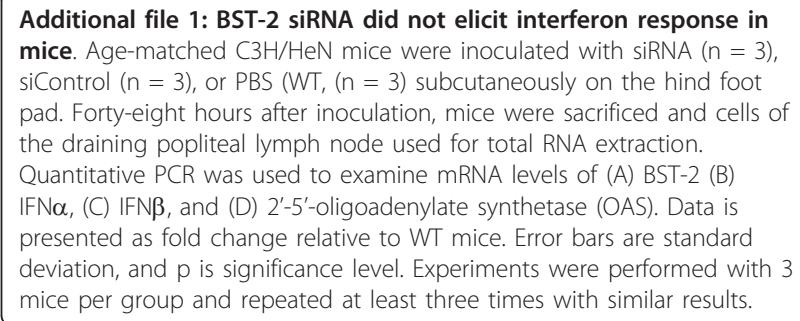

\section{List of abbreviations}

BST-2: bone marrow stromal cell antigen 2; HIV-1: human immunodeficiency virus type 1; HIV-2: human immunodeficiency virus type 2; IFNa: interferon alpha and beta, siRNA: short interfering RNA; shRNA: short hair pin RNA; OAS: oligoadenylate synthetase; NMuMG: normal murine mammary gland; SIV: simian immunodeficiency virus; SEM: scanning electron micrograph; TEM: transmission electron micrograph.

\section{Acknowledgements}

We are also grateful to Dr. Peter Pfeffer of AgResearch Crown Research Institute, New Zealand for the puromycin primer sequence. This research was supported by University of lowa Startup funds to CMO. PHJ was partly supported by University of lowa Startup funds to CMO and Immunology T32 training grant to the University of lowa. This publication was made possible through core services from the University of lowa Vector core and Central Microscopy Research Facility.

\section{Authors' contributions}

CMO conceptualized and designed research, PHJ, HVM, MM, CMO performed research, and analyzed data; PHJ, HVM, MM, RJR, CMO wrote and read the paper, RJR, Contributed reagents. All authors reviewed the manuscript and approved the final version.

\section{Authors' information}

*Department of Microbiology, Carver College of Medicine, University of lowa, lowa City, IA 52242, United States of America.

\section{Competing interests}

The authors declare that they have no competing interests. 
Received: 20 October 2011 Accepted: 27 January 2012

Published: 27 January 2012

\section{References}

1. Neil SJ, Zang T, Bieniasz PD: Tetherin inhibits retrovirus release and is antagonized by HIV-1 Vpu. Nature 2008, 451:425-30.

2. Goffinet C, Schmidt S, Kern C, Oberbremer L, Keppler OT: Endogenous CD317/Tetherin limits replication of HIV-1 and murine leukemia virus in rodent cells and is resistant to antagonists from primate viruses. J Virol 2010, 84:11374-84.

3. Jouvenet N, Neil SJ, Zhadina M, Zang T, Kratovac Z, Lee Y, McNatt M, Hatziioannou T, Bieniasz PD: Broad-spectrum inhibition of retroviral and filoviral particle release by tetherin. J Virol 2009, 83:1837-44.

4. Kaletsky RL, Francica JR, Agrawal-Gamse C, Bates P: Tetherin-mediated restriction of filovirus budding is antagonized by the Ebola glycoprotein Proc Natl Acad Sci USA 2009, 106:2886-91.

5. Lopez LA, Yang SJ, Hauser H, Exline CM, Haworth KG, Oldenburg J, Cannon PM: Ebola virus glycoprotein counteracts BST-2/Tetherin restriction in a sequence-independent manner that does not require tetherin surface removal. J Virol 2010, 84:7243-55.

6. Perez-Caballero D, Zang T, Ebrahimi A, McNatt MW, Gregory DA, Johnson MC, Bieniasz PD: Tetherin inhibits HIV-1 release by directly tethering virions to cells. Cell 2009, 139:499-511.

7. Habermann A, Krijnse-Locker J, Oberwinkler H, Eckhardt M, Homann S, Andrew A, Strebel K, Krausslich HG: CD317/tetherin is enriched in the HIV1 envelope and downregulated from the plasma membrane upon virus infection. J Virol 2010, 84:4646-58.

8. Hammonds J, Wang JJ, Yi H, Spearman P: Immunoelectron microscopic evidence for Tetherin/BST2 as the physical bridge between HIV-1 virions and the plasma membrane. PLoS Pathog 2010, 6:e1000749.

9. Fitzpatrick K, Skasko M, Deerinck TJ, Crum J, Ellisman MH, Guatelli J: Direct restriction of virus release and incorporation of the interferon-induced protein BST-2 into HIV-1 particles. PLoS Pathog 2010, 6:e1000701.

10. Le Tortorec A, Neil SJ: Antagonism to and intracellular sequestration of human tetherin by the human immunodeficiency virus type 2 envelope glycoprotein. J Virol 2009, 83:11966-78.

11. Dube M, Roy BB, Guiot-Guillain P, Mercier J, Binette J, Leung G, Cohen EA: Suppression of Tetherin-restricting activity upon human immunodeficiency virus type 1 particle release correlates with localization of Vpu in the trans-Golgi network. J Virol 2009, 83:4574-90.

12. Goffinet C, Homann S, Ambiel I, Tibroni N, Rupp D, Keppler OT, Fackler OT: Antagonism of $\mathrm{CD} 317$ restriction of human immunodeficiency virus type 1 (HIV-1) particle release and depletion of CD317 are separable activities of HIV-1 Vpu. J Virol 2010, 84:4089-94

13. Hauser H, Lopez LA, Yang SJ, Oldenburg JE, Exline CM, Guatelli JC, Cannon PM: HIV-1 Vpu and HIV-2 Env counteract BST-2/tetherin by sequestration in a perinuclear compartment. Retrovirology 2010, 7:51

14. Pardieu C, Vigan R, Wilson SJ, Calvi A, Zang T, Bieniasz P, Kellam P, Towers GJ, Neil SJ: The RING-CH ligase K5 antagonizes restriction of KSHV and HIV-1 particle release by mediating ubiquitin-dependent endosomal degradation of tetherin. PLOS Pathog 2010, 6:e1000843.

15. Burzyn D, Rassa JC, Kim D, Nepomnaschy I, Ross SR, Piazzon I: Toll-like receptor 4-dependent activation of dendritic cells by a retrovirus. J Virol 2004, 78:576-84

16. Beutner U, Kraus E, Kitamura D, Rajewsky K, Huber BT: B cells are essential for murine mammary tumor virus transmission, but not for presentation of endogenous superantigens. J Exp Med 1994, 179:1457-66.

17. Martin P, Ruiz SR, del Hoyo GM, Anjuere F, Vargas HH, Lopez-Bravo M Ardavin C: Dramatic increase in lymph node dendritic cell number during infection by the mouse mammary tumor virus occurs by a CD62L-dependent blood-borne DC recruitment. Blood 2002, 99:1282-8.

18. Courreges MC, Burzyn D, Nepomnaschy I, Piazzon I, Ross SR: Critical role of dendritic cells in mouse mammary tumor virus in vivo infection. J Virol 2007, 81:3769-77.

19. Dzuris JL, Golovkina TV, Ross SR: Both T and B cells shed infectious mouse mammary tumor virus. J Virol 1997, 71:6044-8.

20. Golovkina TV, Dudley JP, Ross SR: B and T cells are required for mouse mammary tumor virus spread within the mammary gland. $J$ Immuno $1998,161: 2375-82$

21. Okeoma CM, Huegel AL, Lingappa J, Feldman MD, Ross SR: APOBEC3 proteins made in mammary epithelial cells can be packaged into retroviruses and restrict transmission of milk-borne virions. Cell Host Microbe 2010, 8:534-43.

22. Yoo H, Park SH, Ye SK, Kim M: IFN-gamma-induced BST2 mediates monocyte adhesion to human endothelial cells. Cell Immunol 2011 267:23-9.

23. Zhang F, Wilson SJ, Landford WC, Virgen B, Gregory D, Johnson MC, Munch J, Kirchhoff F, Bieniasz PD, Hatziioannou T: Nef proteins from simian immunodeficiency viruses are tetherin antagonists. Cell Host Microbe 2009, 6:54-67.

24. Jolly C, Booth NJ, Neil SJ: Cell-cell spread of human immunodeficiency virus type 1 overcomes tetherin/BST-2-mediated restriction in T cells. $J$ Virol 2010, 84:12185-99.

25. Serra-Moreno R, Jia B, Breed M, Alvarez X, Evans DT: Compensatory changes in the cytoplasmic tail of gp41 confer resistance to tetherin/ BST-2 in a pathogenic nef-deleted SIV. Cell Host Microbe 2011, 9:46-57.

26. Indik S, Gunzburg WH, Salmons B, Rouault F: Mouse mammary tumor virus infects human cells. Cancer Res 2005, 65:6651-9.

27. Gonda MA, Arthur LO, Zeve VH, Fine DL, Nagashima K: Surface localization of virus production on a glucocorticoid-stimulated oncornavirusproducing mouse mammary tumor cell line by scanning electron microscopy. Cancer Res 1976, 36:1084-93.

28. Bittner JJ: SOME POSSIBLE EFFECTS OF NURSING ON THE MAMMARY GLAND TUMOR INCIDENCE IN MICE. Science 1936, 84:162.

29. Shackleford GM, Varmus HE: Construction of a clonable, infectious, and tumorigenic mouse mammary tumor virus provirus and a derivative genetic vector. Proc Natl Acad Sci USA 1988, 85:9655-9.

30. Miesfeld R, Rusconi S, Godowski PJ, Maler BA, Okret S, Wikstrom AC, Gustafsson JA, Yamamoto KR: Genetic complementation of a glucocorticoid receptor deficiency by expression of cloned receptor cDNA. Cell 1986, 46:389-99.

31. Zhang Y, Rassa JC, deObaldia ME, Albritton LM, Ross SR: Identification of the receptor binding domain of the mouse mammary tumor virus envelope protein. J Virol 2003, 77:10468-78.

32. Okeoma CM, Lovsin N, Peterlin BM, Ross SR: APOBEC3 inhibits mouse mammary tumour virus replication in vivo. Nature 2007, 445:927-30.

33. Okeoma CM, Shen M, Ross SR: A novel block to mouse mammary tumor virus infection of lymphocytes in B10.BR mice. J Virol 2008, 82:1314-22.

34. Schliemann C, Roesli C, Kamada H, Borgia B, Fugmann T, Klapper W, Neri D: In vivo biotinylation of the vasculature in B-cell lymphoma identifies BST-2 as a target for antibody-based therapy. Blood 2010, 115:736-44.

35. Parks WP, Ransom JC, Young HA, Scolnick EM: Mammary tumor virus induction by glucocorticoids Characterization of specific transcriptional regulation. J Biol Chem 1975, 250:3330-6.

doi:10.1186/1742-4690-9-10

Cite this article as: Jones et al:: Bone marrow stromal cell antigen 2 (BST-2) restricts mouse mammary tumor virus (MMTV) replication in vivo. Retrovirology 2012 9:10.

\section{Submit your next manuscript to BioMed Central and take full advantage of:}

- Convenient online submission

- Thorough peer review

- No space constraints or color figure charges

- Immediate publication on acceptance

- Inclusion in PubMed, CAS, Scopus and Google Scholar

- Research which is freely available for redistribution

Submit your manuscript at www.biomedcentral.com/submit
C Biomed Central 